\title{
Modelling of Nonlinear Circular Plates Using Modal Analysis: Simulation and Model Validation
}

\author{
J. Juillard, E. Colinet \\ SUPELEC, Dpt. of Measurement, \\ jerome.juillard@supelec.fr
}

\section{Introduction}

There are many micro-structures that can be modelled as simple geometrical elements, such as beams with rectangular cross-sections or circular or rectangular plates. Examples of such structures abound in the MEMS literature [1-2]. Because they are very easy to manipulate, models based upon plate or beam approximations are very interesting. However, they are very often limited to the so-called "small-displacements" regime of the deformable structure, i.e. when the displacements of the structure, say the deflection of the beam or plate, are much smaller than its thickness. In this case, the tensile stresses that are caused by the elongation of the structure can be neglected and this results in purely linear models that can be readily implemented and used. In the "large-displacement" regime, the tensile stresses must be taken into account; this results in nonlinear partial differential equations (PDEs), such as the Von Karman equation for plates, and, as a consequence, in nonlinear models.

The most common way of handling these nonlinear PDEs consists in linearizing them close to a working point, as in [3-4]. This approach can lead to excellent results, provided the linearization hypothesis holds. For sensors/actuators operating over a wide range, as in microswitch applications, this is often not the case and a model valid over the whole range must be found [5-6]. The same goes, for example, for squeeze-film damping for which models that remain valid for large displacements with respect to the gap [7] must be used.

In this paper, a method for modelling the large-displacement actuation of deformable microstructures is proposed and illustrated, in the case of a circular axisymmetric plate. First of all, the problem is formulated and its boundary conditions are expressed as functions of the two unknowns, displacements $w$ and Airy stress function $F$. The problem is then split in two parts, each part corresponding to one equation of Von Karman, to which the techniques of modal analysis are applied in order to obtain a set of ordinary differential equations (ODEs). The implementation of the resulting model is then discussed and some simulation results are given, as a basis for comparison.

\section{Problem formulation and methodology}

\section{Problem formulation}

Let us consider a plate with thickness $h$, radius $R_{0}$, Young's modulus $E$ and Poisson's coefficient $v$ and density $\rho$. The displacements $w$ at any point of this plate, and the internal stresses, can be expressed as a function of applied pressure $P$, thanks to the equations of Von Karman (1) [8].

$$
\left\{\begin{array}{l}
\frac{E h^{3}}{12\left(1-v^{2}\right)} \Delta^{2} w+\rho h \frac{\partial^{2} w}{\partial t^{2}}=P(x, y, t)+h\left(\frac{\partial^{2} F}{\partial y^{2}} \frac{\partial^{2} w}{\partial x^{2}}+\frac{\partial^{2} F}{\partial x^{2}} \frac{\partial^{2} w}{\partial y^{2}}-2 \frac{\partial^{2} F}{\partial x \partial y} \frac{\partial^{2} w}{\partial x \partial y}\right) \\
\Delta^{2} F=-E\left(\frac{\partial^{2} w}{\partial x^{2}} \frac{\partial^{2} w}{\partial y^{2}}-\left(\frac{\partial^{2} w}{\partial x \partial y}\right)^{2}\right)
\end{array}\right.
$$

$F$ is known as the Airy stress function. The internal stresses can be expressed as second-order derivatives of $F$. 
For an axisymmetric circular membrane, (1) can be written:

$$
\left\{\begin{array}{l}
\frac{E h^{3}}{12\left(1-v^{2}\right)} \Delta^{2} w+\rho h \frac{\partial^{2} w}{\partial t^{2}}=P(r, t)+\frac{h}{r}\left(\frac{\partial^{2} F}{\partial r^{2}} \frac{\partial w}{\partial r}+\frac{\partial F}{\partial r} \frac{\partial^{2} w}{\partial r^{2}}\right) \\
\Delta^{2} F=-\frac{E}{r} \frac{\partial w}{\partial r} \frac{\partial^{2} w}{\partial r^{2}}
\end{array}\right.
$$

As for the boundary conditions, considering the membrane is clamped on its edge, we may write:

$$
\left\{\begin{array}{l}
u\left(R_{0}\right)=v\left(R_{0}\right)=0 \\
w\left(R_{0}\right)=\left.\frac{\partial w}{\partial r}\right|_{r=R_{0}}=0
\end{array}\right.
$$

where $u$ and $v$ are the transverse (i.e. radial and ortho-radial) displacements of the membrane. In the axisymmetric case, only $u$ is non zero and it becomes simple to express the boundary condition on the radial displacements in terms of the Airy stress function:

$u\left(R_{0}\right)=\frac{1}{E}\left(\left.v \frac{\partial F}{\partial r}\right|_{R_{0}}-\left.R_{0} \frac{\partial^{2} F}{\partial r^{2}}\right|_{R_{0}}\right)=0$

In order to solve the second equation of Von Karman, a second boundary condition must be found. Since only the derivatives of the Airy stress function have physical significance, we choose to impose:

$F\left(R_{0}\right)=0$

\section{Methodology}

In order to obtain a set of ODEs from equations (2), the problem is split into two parts, each corresponding to a line of (2). The coupled nonlinear problem is then expanded into two linear problems, with "nonlinear" source terms. A variety of methods can be employed in order to solve either of these problems (such as finite differences or finite elements), to which we prefer modal analysis, which, in this simple geometry, leads to analytical expressions for the mode shapes. Using a Galerkin procedure, each linear PDE can then be transformed into a system of linear ODEs with nonlinear source-terms. This can be summed up in the following formulas. Let $\Lambda_{\vec{x}}$ be a spatial linear differential operator and $\Psi_{t}$ be a time linear differential operator. From any linear PDE:

$\Lambda_{\vec{x}} \varphi+\Psi_{t} \varphi=f$

with homogenous ${ }^{1}$ boundary conditions on $\delta \Omega$, one can deduce an equivalent infinite set of ODEs. One method for obtaining such a set is using modal analysis, i.e. writing:

$\varphi=\sum_{k=1}^{\infty} a_{k}(t) \varphi_{k}(\vec{x})$

and imposing that each $\varphi_{k}(\vec{x})$ be an eigenmode of operator $\Lambda_{\vec{x}}$ :

$\Lambda_{\vec{x}} \varphi_{k}(\vec{x})=\lambda_{k} \varphi_{k}(\vec{x})$

with the same boundary conditions as $\varphi^{2}$. The eigenmodes can be chosen so that they form an orthonormal basis of the solution space of (6). In that case, injecting (7) in (6) and projecting on the $\varphi_{k}(\vec{x})$ - the Galerkin procedure - one finally obtains:

$\lambda_{k} a_{k}+\Psi_{t} a_{k}=\left\langle f \mid \varphi_{k}\right\rangle_{\Omega}, \forall k \in \mathbf{N}$

System (9) is then truncated so that it can practically be solved. This method can be used in many problems, its major difficulty lies in solving (8) to obtain the $\varphi_{k}(\vec{x})$; this can be done

\footnotetext{
${ }^{1}$ There are cases when this condition is not strictly necessary. However, for the clarity and the brevity of this paper, we prefer not to go into further detail.

${ }^{2}$ In the case of a PDE with boundary conditions at the infinite, the eigenmodes are no longer a discrete set but a continuum.
} 
analytically in simple geometrical cases (see [9]). For more complex geometries, numerical methods, such as the finite element method, may be used to compute the eigenmodes and the eigenvalues of a PDE.

\section{Modal analysis of the first equation of Von Karman}

The modal analysis of the linear part of the first equation of Von Karman is well-known [910], in the clamped circular case. Writing displacement $w(r, t)$ as the weighted sum of modal components $w_{k}(r)$ :

$$
w(r, t)=\sum_{k=1}^{N} a_{k}(t) w_{k}(r)
$$

the mode shapes, in the axisymmetric case, are:

$w_{k}(r)=A_{k}\left(I_{0}\left(\alpha_{k} R_{0}\right) J_{0}\left(\alpha_{k} r\right)-J_{0}\left(\alpha_{k} R_{0}\right) I_{0}\left(\alpha_{k} r\right)\right)(11)$

where $\alpha_{k}$ is a solution of:

$I_{0}\left(\alpha_{k} R_{0}\right) J_{1}\left(\alpha_{k} R_{0}\right)+J_{0}\left(\alpha_{k} R_{0}\right) I_{1}\left(\alpha_{k} R_{0}\right)=0$

and $A_{k}$ can be chosen so that the mode shapes form an orthonormal basis with respect to the following scalar product:

$$
\langle f \mid g\rangle=\int_{0}^{R_{0}} r f(r) g(r) d r
$$

In the more general non-axisymmetric case, Bessel functions of integer order larger than zero and simple trigonometric functions appear in the formula of the mode shapes. These can be found in [10].

Knowing the value of the Airy stress function and of its derivatives, the first equation of Von Karman is simplified into a set of ordinary differential equations (ODEs). Thus, one can transform:

$\frac{E h^{3}}{12\left(1-v^{2}\right)} \Delta^{2} w+\rho h \frac{\partial^{2} w}{\partial t^{2}}=P(r, t)+\frac{h}{r}\left(\frac{\partial^{2} F}{\partial r^{2}} \frac{\partial w}{\partial r}+\frac{\partial F}{\partial r} \frac{\partial^{2} w}{\partial r^{2}}\right)=P(r, t)+\Phi(r, t)$

into the following set of ODEs (14), by:

- going into the Laplace domain - with Laplace parameter $s$,

- using the Galerkin procedure, i.e. by:

$\circ$ writing $w(r)$ in the basis formed by the eigenmodes (11),

$\circ$ projecting the resulting equation on the basis functions.

Provided coefficients $A_{k}$ have been chosen appropriately, one obtains:

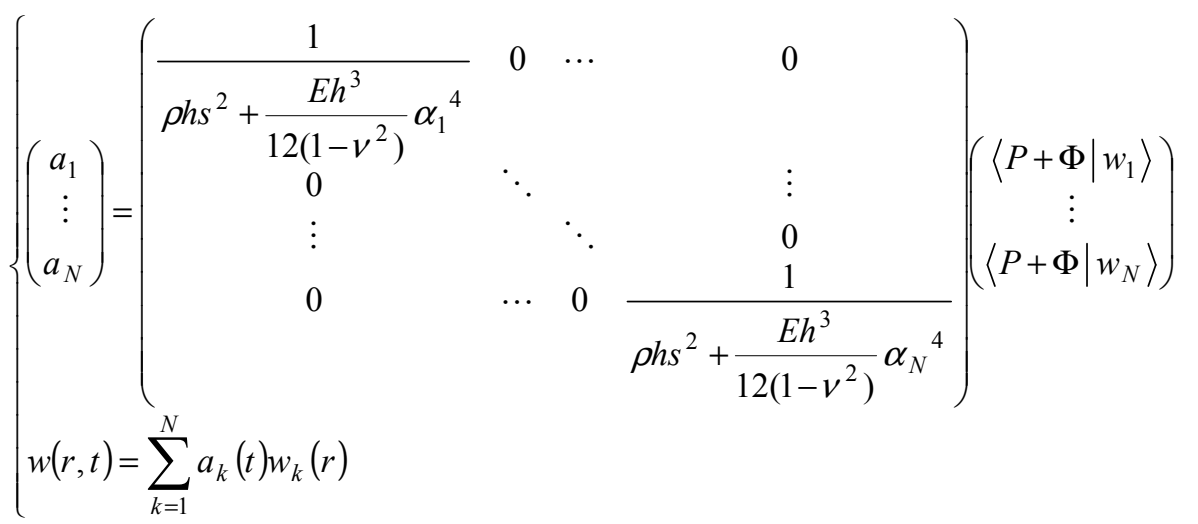

It must be noted that, in the case when residual stress is present in the plate, the same approach, with the same displacement mode shapes, may be used. In the presence of residual stress $\sigma$, the first equation of Von Karman is: 
$\frac{E h^{3}}{12\left(1-v^{2}\right)} \Delta^{2} w+\sigma \Delta w+\rho h \frac{\partial^{2} w}{\partial t^{2}}=P(r, t)+\Phi(r, t)$

Now, this could be treated exactly as above, by calculating the eigenmodes of operator $\frac{E h^{3}}{12\left(1-v^{2}\right)} \Delta^{2}()+.\sigma \Delta($.$) and this would lead to expressions of the mode shapes. However, for large$ values of $\sigma$, the fourth-order operator degenerates to a second-order operator and this may result in numerical problems for evaluating its eigenvalues. An efficient way of solving this problem is then to use the eigenmodes and eigenvalues obtained through (11) and (12) for the biharmonic operator. The first equation of Von Karman (15) then becomes:

$$
\left\{\begin{array}{c}
\left(\begin{array}{c}
a_{1} \\
\vdots \\
a_{N}
\end{array}\right)=\left(\begin{array}{cccc}
\frac{1}{\rho h s^{2}+\frac{E h^{3}}{12\left(1-v^{2}\right)} \alpha_{1}^{4}} & 0 & \cdots & 0 \\
0 & \ddots & & \vdots \\
\vdots & & \ddots & 0 \\
0 & \cdots & 0 & \frac{1}{\rho s^{2}+\frac{E h^{3}}{12\left(1-v^{2}\right)} \alpha_{N}^{4}}
\end{array}\right)\left(\left(\begin{array}{c}
\left\langle P+\Phi \mid w_{1}\right\rangle \\
\vdots \\
\left\langle P+\Phi \mid w_{N}\right\rangle
\end{array}\right)-\sigma\left(\begin{array}{ccc}
\left\langle\Delta w_{1} \mid w_{1}\right\rangle & \cdots & \left\langle\Delta w_{N} \mid w_{1}\right\rangle \\
\vdots & \ddots & \vdots \\
\left\langle\Delta w_{1} \mid w_{N}\right\rangle & \cdots & \left\langle\Delta w_{N} \mid w_{N}\right\rangle
\end{array}\right)\left(\begin{array}{c}
a_{1} \\
\vdots \\
a_{N}
\end{array}\right)\right) \\
w(r, t)=\sum_{k=1}^{N} a_{k}(t) w_{k}(r)
\end{array}\right.
$$

For a given number of modal components, the (symmetric) matrix on the right-hand side can be evaluated once and for all. One can then solve for the modal coefficients by implementing the second-term on the right hand side as a feedback term (cf. fig. 1).

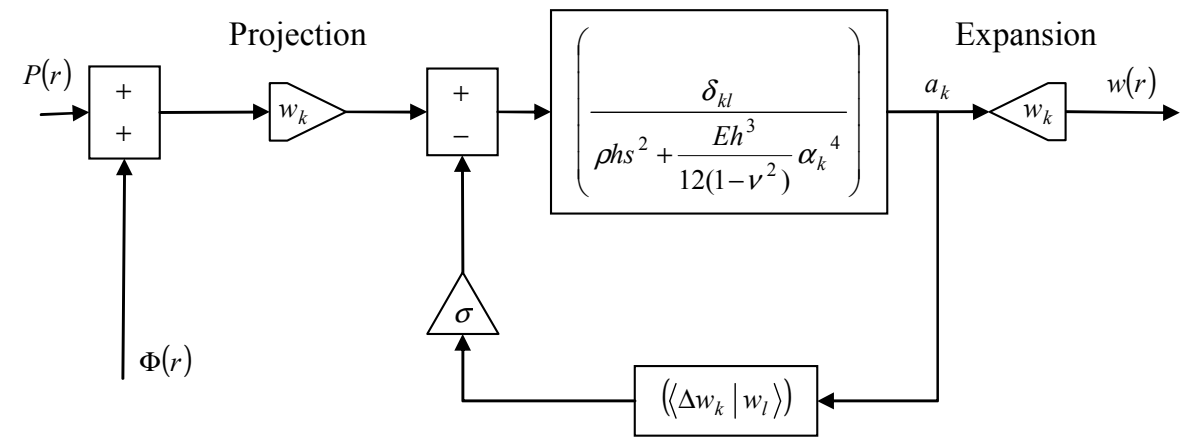

fig. 1: block-diagram representation of (16).

\section{Modal analysis of the second equation of Von Karman}

Let us now consider the linear part of the second equation of Von Karman. There exist in the literature some efforts to handle the equations of Von Karman through modal analysis [6]. The present approach is almost similar to that of [11]. However, in [11] the authors choose to transform the second equation of Von Karman and solve for the derivative of the Airy stress function. In the present work, we directly solve for the Airy stress function: this yields closedform expressions for the eigenmodes of the Airy stress function. Although the two approaches should eventually give the same results, they are not equivalent from a numerical point of view, as we will show further on.

Let us expand the Airy stress function into a sum of modal components:

$$
F(r, t)=\sum_{k=1}^{N} b_{k}(t) F_{k}(r)
$$

with 


$$
\left\{\begin{array}{l}
\Delta^{2} F_{k}=\beta_{k}{ }^{4} F_{k} \\
F_{k}\left(R_{0}\right)=0 \\
\left.v \frac{\partial F_{k}}{\partial r}\right|_{R_{0}}-\left.R_{0} \frac{\partial^{2} F_{k}}{\partial r^{2}}\right|_{R_{0}}=0
\end{array}\right.
$$

The general form of the solution is:

$F_{k}(r)=B_{k}\left(I_{0}\left(\beta_{k} R_{0}\right) J_{0}\left(\beta_{k} r\right)-J_{0}\left(\beta_{k} R_{0}\right) I_{0}\left(\beta_{k} r\right)\right)(19)$

where $\beta_{k}$ is the $k^{\text {th }}$ non-trivial solution of:

$\Lambda_{v, R_{0}}(\beta)=(v+1)\left(J_{1}\left(\beta R_{0}\right)+J_{0}\left(\beta R_{0}\right) \frac{I_{1}\left(\beta R_{0}\right)}{I_{0}\left(\beta R_{0}\right)}\right)-2 \beta R_{0} J_{0}\left(\beta R_{0}\right)=0$

$\Lambda_{v, R_{0}}(\beta)$ is plotted in fig. 2 for different values of Poisson's coefficient.

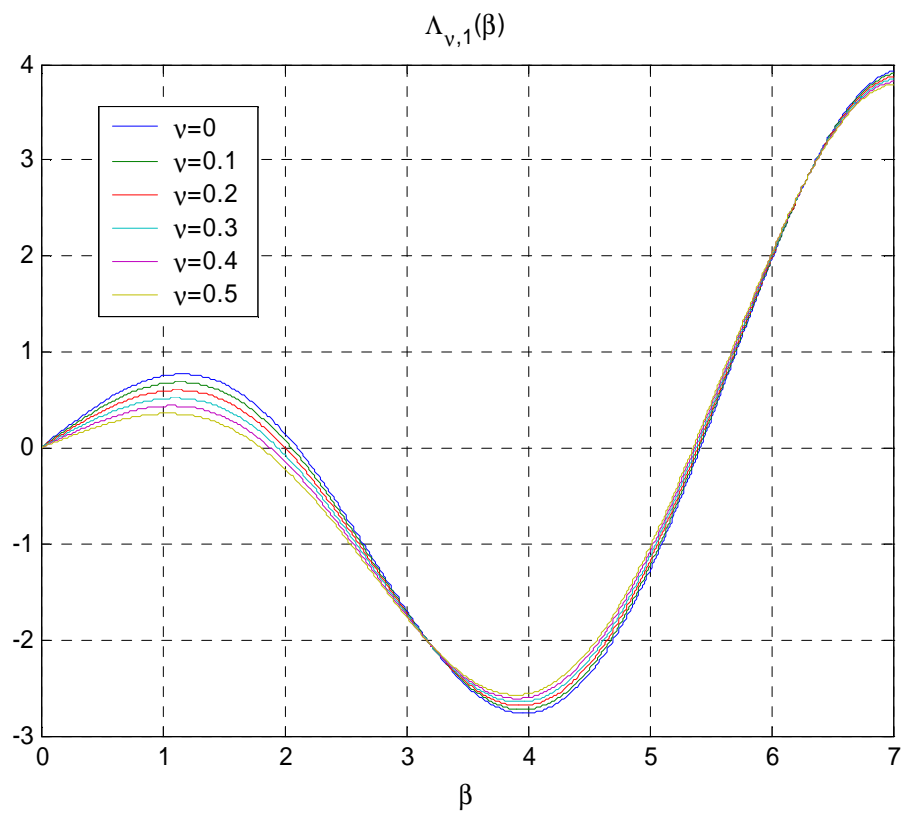

fig. 2: $\Lambda_{v, R_{0}}(\beta)$ for different values of Poisson's coefficient and $R_{0}=1$.

For large mode numbers, the mode shapes do not differ much, because the second term of (20) dominates as $\beta$ increases. The first two modes of the Airy stress function are represented in fig. 3 , for different values of Poisson's coefficient.
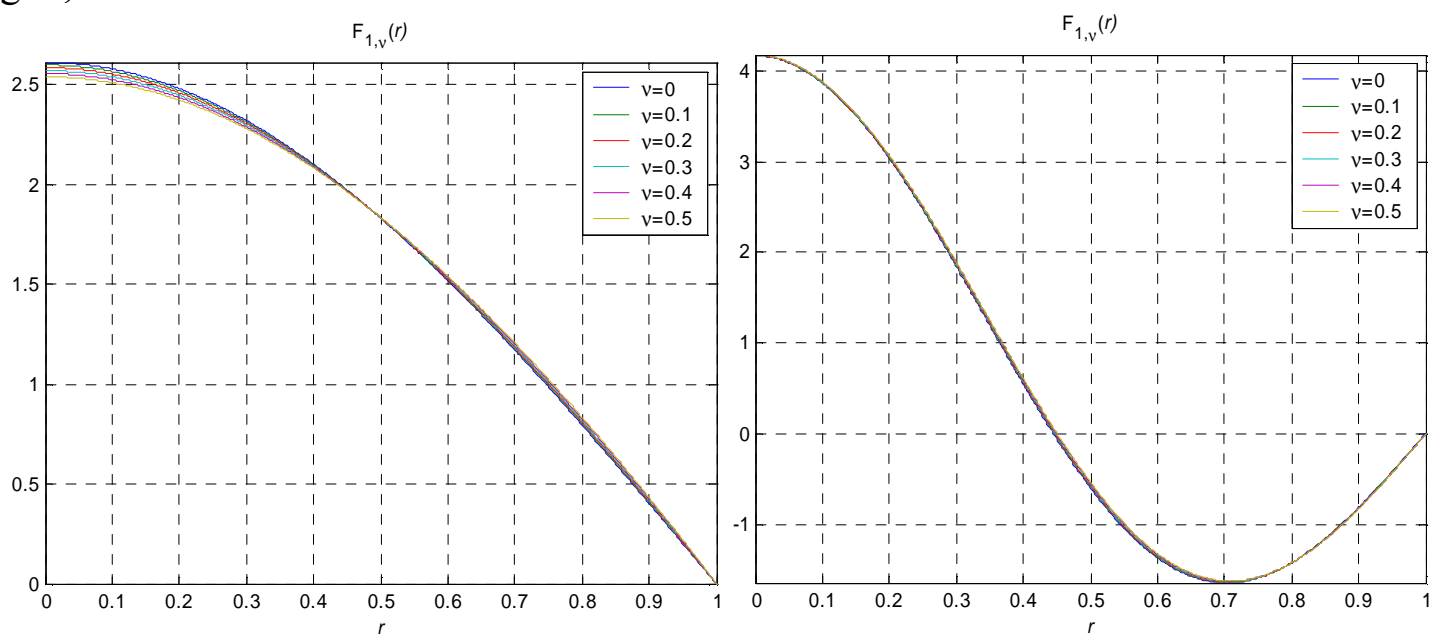

fig. 3: first two mode shapes of the Airy stress function for different values of Poisson's coefficient. Coefficients $B_{k}$ have been chosen so that the mode shapes are orthonormal. 


\begin{tabular}{|c|c|c|c|}
\hline & $1^{\text {st }}$ mode & $2^{\text {nd }}$ mode & $3^{\text {rd }}$ mode \\
\hline$\nu=0$ & 2.1079881249672 & 5.4188347079883 & 8.5920247747293 \\
\hline$\nu=0.1$ & 2.0624874640420 & 5.4074025423301 & 8.5853967720548 \\
\hline$\nu=0.2$ & 2.0118601470532 & 5.3957032410861 & 8.5786801978157 \\
\hline$\nu=0.3$ & 1.9549127826748 & 5.3837287263695 & 8.5718736616392 \\
\hline$\nu=0.4$ & 1.8899582175831 & 5.3714706889439 & 8.5649757586549 \\
\hline$v=0.5$ & 1.8144846969537 & 5.3589205900056 & 8.5579850701462 \\
\hline
\end{tabular}

Table 1: values of $\beta_{k}$ vs. Poisson's coefficient, for the first three modes.

Finally, one must note that these eigenmodes are orthogonal with respect to the scalar product (13) and that, provided $B_{k}$ is appropriately chosen, the mode shapes can be made orthonormal.

Thus, knowing the value of the displacements, one can compute the value of the Airy stress function as the solution of:

$\Delta^{2} F=-\frac{E}{r} \frac{\partial w}{\partial r} \frac{\partial^{2} w}{\partial r^{2}}$

which can be simplified into

$$
\left\{\begin{array}{l}
\left(\begin{array}{c}
b_{1} \\
\vdots \\
b_{M}
\end{array}\right)=\left(\begin{array}{cccc}
\frac{1}{\beta_{1}{ }^{4}} & 0 & \cdots & 0 \\
0 & \ddots & & \vdots \\
\vdots & & \ddots & 0 \\
0 & \cdots & 0 & \frac{1}{\beta_{1}{ }^{M}}
\end{array}\right)\left(\left\langle-\frac{E}{r} \frac{\partial w}{\partial r} \frac{\partial^{2} w}{\partial r^{2}} \mid F_{1}\right\rangle\right. \\
\vdots \\
F(r, t)=\sum_{k=1}^{M} b_{k}(t) F_{k}(r)
\end{array}\right.
$$

The block diagram corresponding to the calculation of the source-term $\Phi$ of (15) is shown in fig. 4.

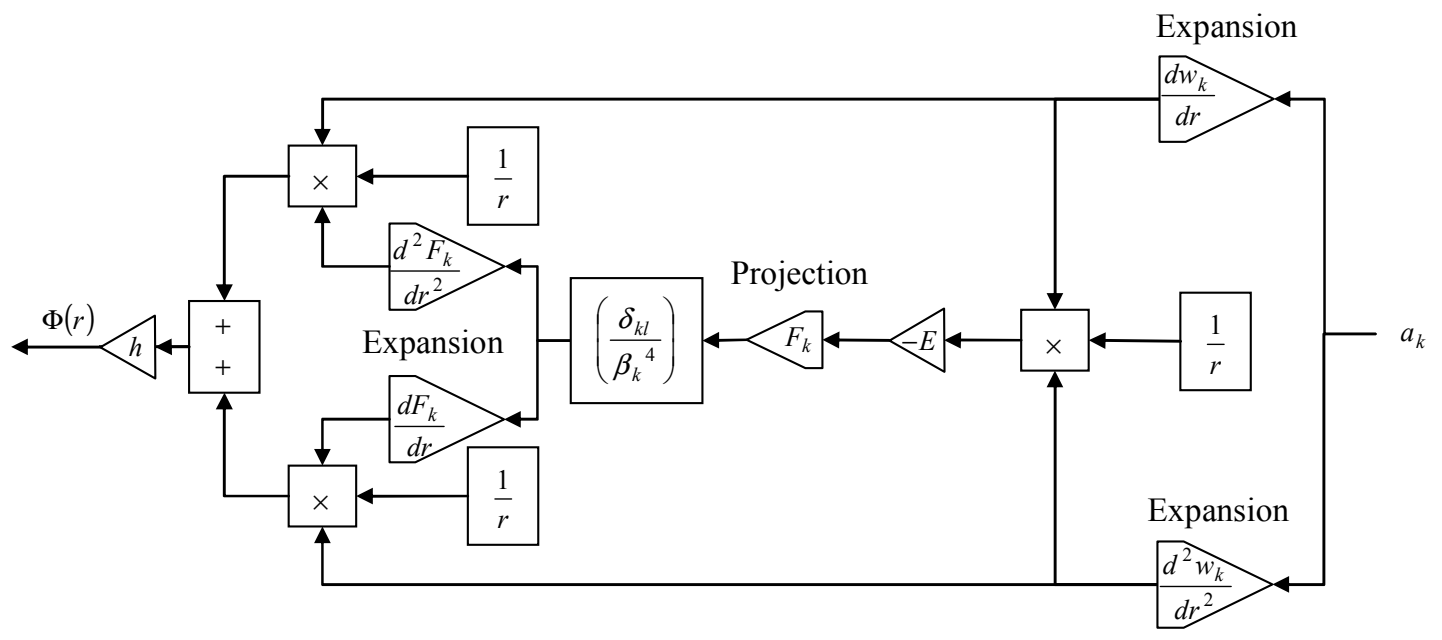

fig. 4: calculation of $\Phi$ from the modal coefficients $a_{k}$.

\section{Implementation}

We have shown in the last two parts how the equations of Von Karman could be simplified into: 
- a set of second-order ODEs with a nonlinear source term - (14) or (16) - for the first equation of Von Karman,

- a set of equalities (22), for the second equation of Von Karman, from which the source term of (14) or (16) can be easily calculated.

Now, this may be easily implemented using any ODE solver, such as those provided in Matlab/Simulink. Stiff solvers [12] should be preferred to non-stiff ones, because of the presence of very different time-scales in (14) or (16) and also because of the possible presence of sharp changes in the solution due to the nonlinear nature of the problem. The derivatives appearing in the expansions of fig. 4 or are analytically calculated. It must be noted that many scalar products can be numerically evaluated once and for all at the beginning of the simulation. One must note that the computational effort of evaluating these scalar products is dependent on the eigenmodes chosen for the modal analysis of Von Karman's second equation. When using a polynomial quadrature scheme, the integrals appearing in formula (7.6.66) of [11] require a much finer discretization than those appearing in expression (21). For example, in the neighbourhood of $r=0$, the integrands of (21) behave as first-order polynomials, whereas those of [11] behave as third-order polynomials (fig. 5).

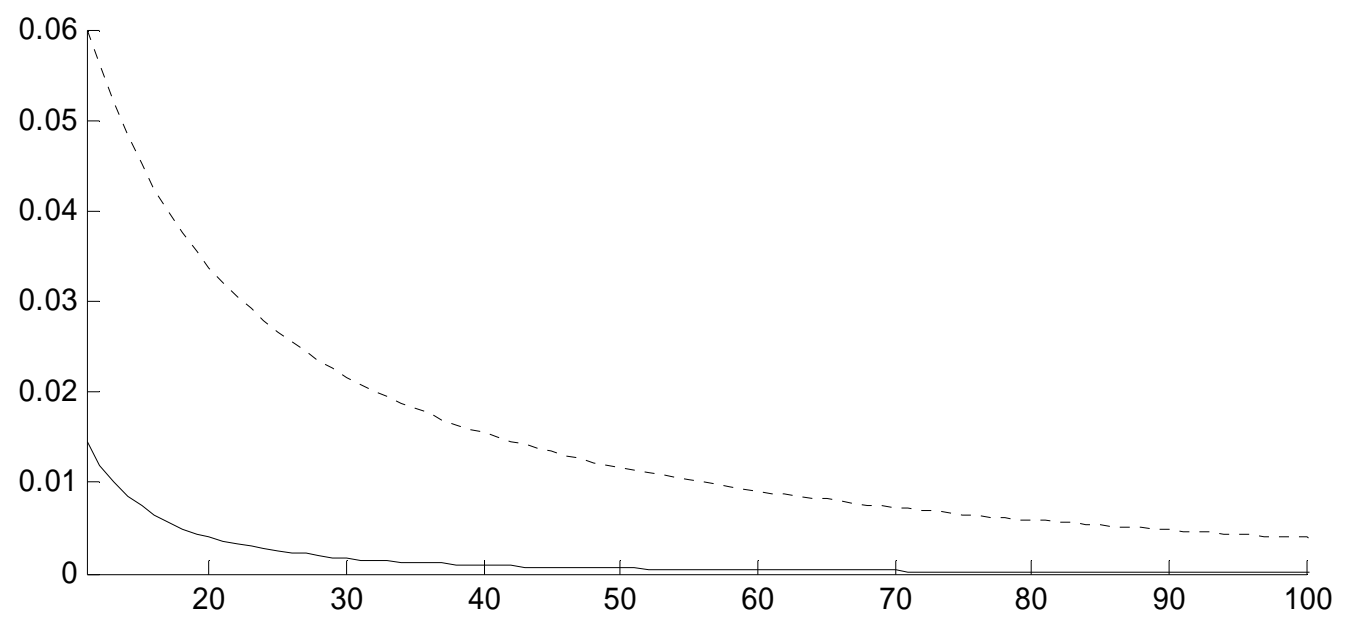

fig. 5: relative error as a function of number of quadrature points for the first integrals appearing in (21) - $\int_{0}^{R_{0}} \frac{\partial w_{1}}{\partial r} \frac{\partial^{2} w_{1}}{\partial r^{2}} F_{1}(r) d r$, continuous line - and in [11] - $\int_{0}^{R_{0}} \frac{\partial w_{1}}{\partial r} \frac{\partial w_{1}}{\partial r} J_{1}\left(\varsigma_{k} r\right) d r$, dashed line. The quadrature is a first-order Newton-Cotes scheme.

The number $N$ of displacement modes should be chosen so that:

- the forcing term in (14) or (16) can be accurately represented using these $N$ modes,

- the natural frequency of the $N^{\text {th }}$ mode is larger than the frequency of the forcing term. As for the minimal number $M$ of Airy stress function modes, it should be chosen larger than $N$, so that the forcing term of (22) may also be accurately represented. In all the tested configurations, choosing $M$ larger than $N$ did not result in any significant change in the solution of the problem, nor did it affect much the simulation speed.

However, choosing too small a number of modes can have a drastic impact on a simulation result. For example, consider a circular plate with the following characteristics:

$R_{0}=5.10^{-3} \mathrm{~m}, h=1.5 \times 10^{-6} \mathrm{~m}, E=170 \mathrm{GPa}, \nu=0.2, \rho=2320 \mathrm{~kg} \cdot \mathrm{m}^{-3}$.

The damping coefficient $c$ is set to $1 \mathrm{~kg} \cdot \mathrm{m}^{-2} \cdot \mathrm{s}^{-1}$.

The system's input is a uniform pressure distribution $P=P_{0} \sin (\omega t)$, with $P_{0}=10^{-1} \mathrm{~Pa}$ and $\omega=5.69 \times 10^{3}$ rad.s ${ }^{-1}$, which is close to the natural pulsation of the second displacement mode. 


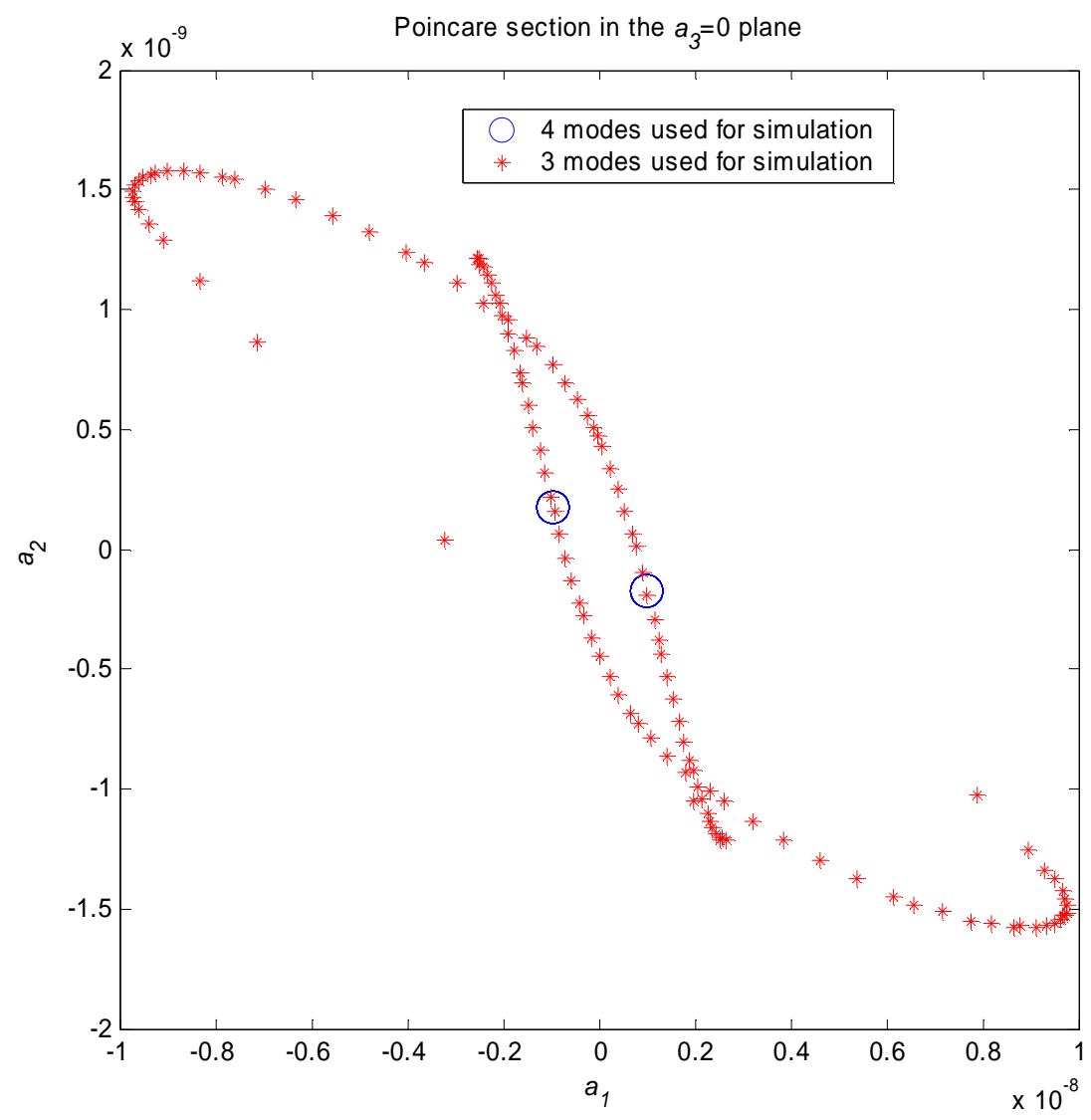

fig. 6: Poincaré section in the $a_{3}=0$ plane for different simulation conditions.

Fig. 6 shows a Poincaré section of the system when a varying number of displacement modes is taken into account in the simulation. The corresponding phase space is the space of modal coordinates $\left(a_{1}, a_{2}, a_{3}\right)$. When only three displacement modes are taken into account, the system has a quasi-periodic motion in phase-space, no matter what value the tolerance of the simulation algorithm is set to. When taking into account four - or more - displacement modes, the motion of the system becomes periodic: the Poincare section is then reduced to two points.

\section{Model validation}

In this section, our model is validated from a mathematical point of view thanks to an analytical solution of the equations of Von Karman. This solution can be obtained by an approach similar to that in [16]: instead of solving Von Karman's equations for the displacements, we find a solution to the inverse problem, i.e. from a given set of displacements we find the corresponding pressure distribution.

Let us then consider a displacement of the following form:

$w(r, t)=W_{0}\left(1-\frac{r^{2}}{R_{0}^{2}}\right)^{2} T(t)$

In order to solve for the pressure, one starts by solving Von Karman's second equation:

$\Delta^{2} F=-\frac{E}{r} \frac{\partial w}{\partial r} \frac{\partial^{2} w}{\partial r^{2}}=-\frac{16 E W_{0}^{2}}{R_{0}^{4}}\left(1-4 \frac{r^{2}}{R_{0}^{2}}+3 \frac{r^{4}}{R_{0}^{4}}\right) T(t)^{2}$

Using boundary condition (4), one obtains:

$F(r, t)=-E W_{0}^{2}\left(\frac{1}{48} \frac{r^{8}}{R_{0}^{8}}-\frac{1}{9} \frac{r^{6}}{R_{0}^{6}}+\frac{1}{4} \frac{r^{4}}{R_{0}^{4}}-\frac{1}{12} \frac{5-3 v}{1-v} \frac{r^{2}}{R_{0}^{2}}\right) T(t)^{2}$ 
This must then be input into Von Karman's first equation. The pressure corresponding to (22) then comes out as:

$$
\begin{aligned}
& P(r, t)=\frac{E h^{3}}{12\left(1-v^{2}\right)} \Delta^{2} w+c \frac{\partial w}{\partial t}+\rho h \frac{\partial^{2} w}{\partial t^{2}}-\frac{h}{r}\left(\frac{\partial^{2} F}{\partial r^{2}} \frac{\partial w}{\partial r}+\frac{\partial F}{\partial r} \frac{\partial^{2} w}{\partial r^{2}}\right)= \\
& W_{0}\left(\frac{16}{3\left(1-v^{2}\right)} \frac{E h^{3}}{R_{0}^{4}} T+c\left(1-\frac{r^{2}}{R_{0}^{2}}\right)^{2} \dot{T}+\rho h\left(1-\frac{r^{2}}{R_{0}^{2}}\right)^{2} \ddot{T}\right) \\
& +\frac{4}{3} \frac{E h W_{0}^{3}}{R_{0}^{4}}\left(\frac{5-3 v}{1-v}-\frac{22-18 v}{1-v} \frac{r^{2}}{R_{0}^{2}}+30 \frac{r^{4}}{R_{0}^{4}}-20 \frac{r^{6}}{R_{0}^{6}}+5 \frac{r^{8}}{R_{0}^{8}}\right) T^{3}
\end{aligned}
$$

Note that this approach can be used for any displacement field of the form (26) provided the boundary conditions, initial conditions and smoothness conditions are met.

$w(r, t)=R(r) T(t)$

It can also be extended without much difficulty to systems involving squeeze film damping and electrostatic actuation such as is common in MEMS, as well as other types of displacement-dependent nonlinearities. For example, it is clear that in (25) the damping coefficient can depend on $w$ : the pressure distribution will not be any harder to compute. Supposing that the pressure is displacement-dependent and follows (27), as in the case of electrostatic actuation:

$$
P(r, t)=C \frac{\Theta(r, t)}{(G-w(r, t))^{2}}
$$

one can then easily use the same approach as above to solve for $\Theta(r, t)$.

Thus, this approach provides us with a simple, exact analytical way of testing and - hopefully - validating reduced-order MEMS models involving nonlinearities: for a given displacement shape, such as (22), the corresponding pressure is calculated using (25). This pressure can then be input into a reduced-order model of the plate. The error can then be computed by subtracting the model's output to the initial displacements (22) and integrating with respect to time and space. For example, we plot in fig. 7, the error between the analytical and the numerical solution when:

$$
\begin{aligned}
& T(t)=1-\cos (\omega t), \omega=100 \mathrm{rad} . \mathrm{s}^{-1} \\
& c=\frac{D}{(G-w(r, t))^{3}}, D=10^{-13} \mathrm{~kg} \cdot \mathrm{m} \cdot \mathrm{s}^{-1}, W_{0}=2.25 \times 10^{-6} \mathrm{~m} .
\end{aligned}
$$

The nonlinear damping is taken into account as a nonlinear source term in the model of fig. 1 . The displacements are large with respect to the plate's thickness and also with respect to the gap.
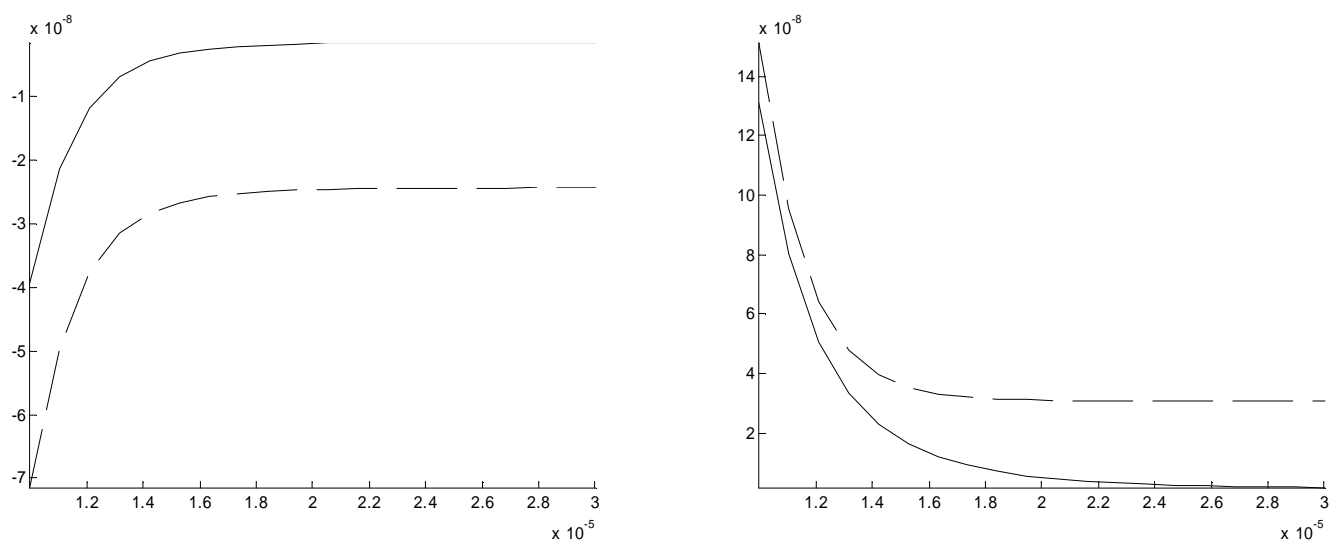

fig. 7: mean value (left) and standard deviation (right) of the error between analytical model and numerical model as a function of gap size. The continuous lines correspond to the present model, the dashed lines to a similar model using the basis functions of [11], with the same simulation parameters. 
Figure 7 shows that both models are valid, even for moderately small values of the gap. However, our model yields slightly more accurate results for the same computational cost. This must not be neglected in a context of MEMS design: for example, design approaches based on Monte-Carlo methods rely on a very large number of simulations, for which the computational cost must be as small as possible. The same is true for model-based parameter identification methods. Finally, this discrepancy between the two models might also be amplified in the case of a pull-in voltage analysis.

\section{Results}

In this section, some results are given for reference. These results have been obtained for the following set of plate parameters: $R_{0}=5 \times 10^{-3} \mathrm{~m}, h=1,5 \times 10^{-6} \mathrm{~m}, E=170 \mathrm{GPa}, \nu=0.2, \rho=2320$ $\mathrm{kg} \cdot \mathrm{m}^{-3}$. The damping coefficient $c$ is set to $1 \mathrm{~kg} \cdot \mathrm{m}^{-2} \cdot \mathrm{s}^{-1}$.

The system's input is a uniform pressure distribution $P=P_{0} \sin (\omega t)$, with varying $P_{0}$ and $\omega$. Throughout the following examples, the number of displacement modes and the number of Airy stress function modes is chosen equal to 6 .

The tool used for running these simulations is Matlab/Simulink, with the ode23tb ODEsolver. All solver parameters are set to default, except for the algorithm's absolute tolerance which is set to $10^{-12}$.

The results obtained with our model are also compared to the experimental data in [13].

\section{Influence of pressure intensity}

According to [8], increasing the input pressure intensity should result in a stiffening of the membrane and, thus, to a shift of its natural frequency. This phenomenon is illustrated in fig. 8.
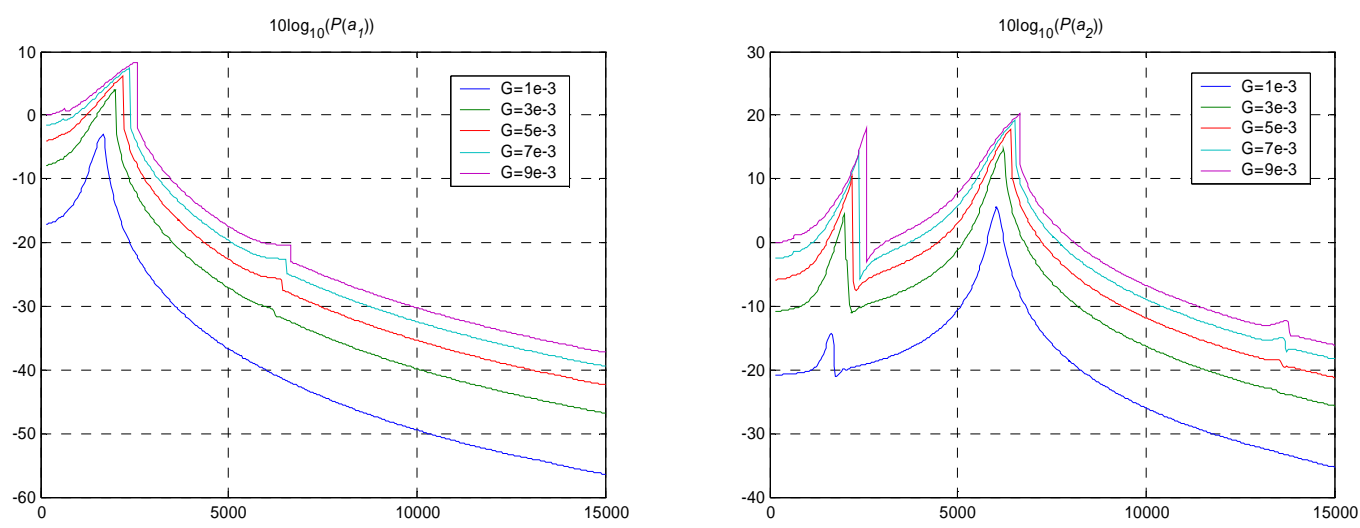

fig. 8: frequency responses for the first (left) and for the second (right) mode for different values of the input pressure.

For larger excitations, jumps in the frequency response curves should appear i.e., for a given excitation frequency, more than one oscillation state can be reached, depending on the system's initial conditions. This is illustrated in the next section.

\section{Influence of initial conditions}

For increasing values of the pressure intensity, one expects to see jumps (bifurcations) appearing in the frequency response curve. These jumps define frequency domains in which more than one oscillation state can be reached. For example, using $P_{0}=10^{-2} \mathrm{~Pa}$, any of the frequency responses of fig. 9 can be obtained, depending on the system's initial conditions.

It is interesting to note that there exists a small region in which three different oscillation states are possible for one excitation frequency. We have plotted in fig. 10 the attraction basins of these oscillation states, i.e. the set of points in the reduced state-space $\left(a_{1}, a_{2}\right)$ such 
that initial conditions chosen in this set dynamically evolve to a given oscillation state (from MathWorld [14]).
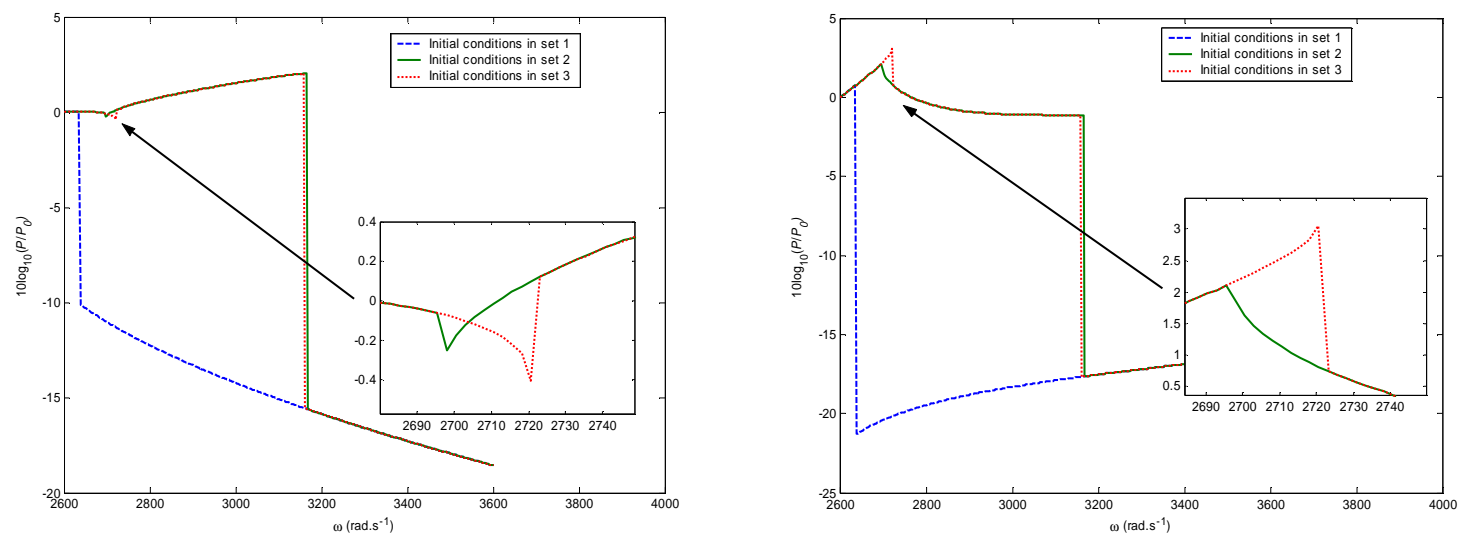

fig. 9: frequency responses for the first (left) and for the second (right) mode for different values of the input pressure and different initial conditions.

These results were obtained rather uneconomically through direct simulation of the set of nonlinear ODEs (13), by sweeping the space of initial conditions. A more reasonable approach for obtaining the different oscillation states would be, for instance, to use harmonic balance techniques [15].

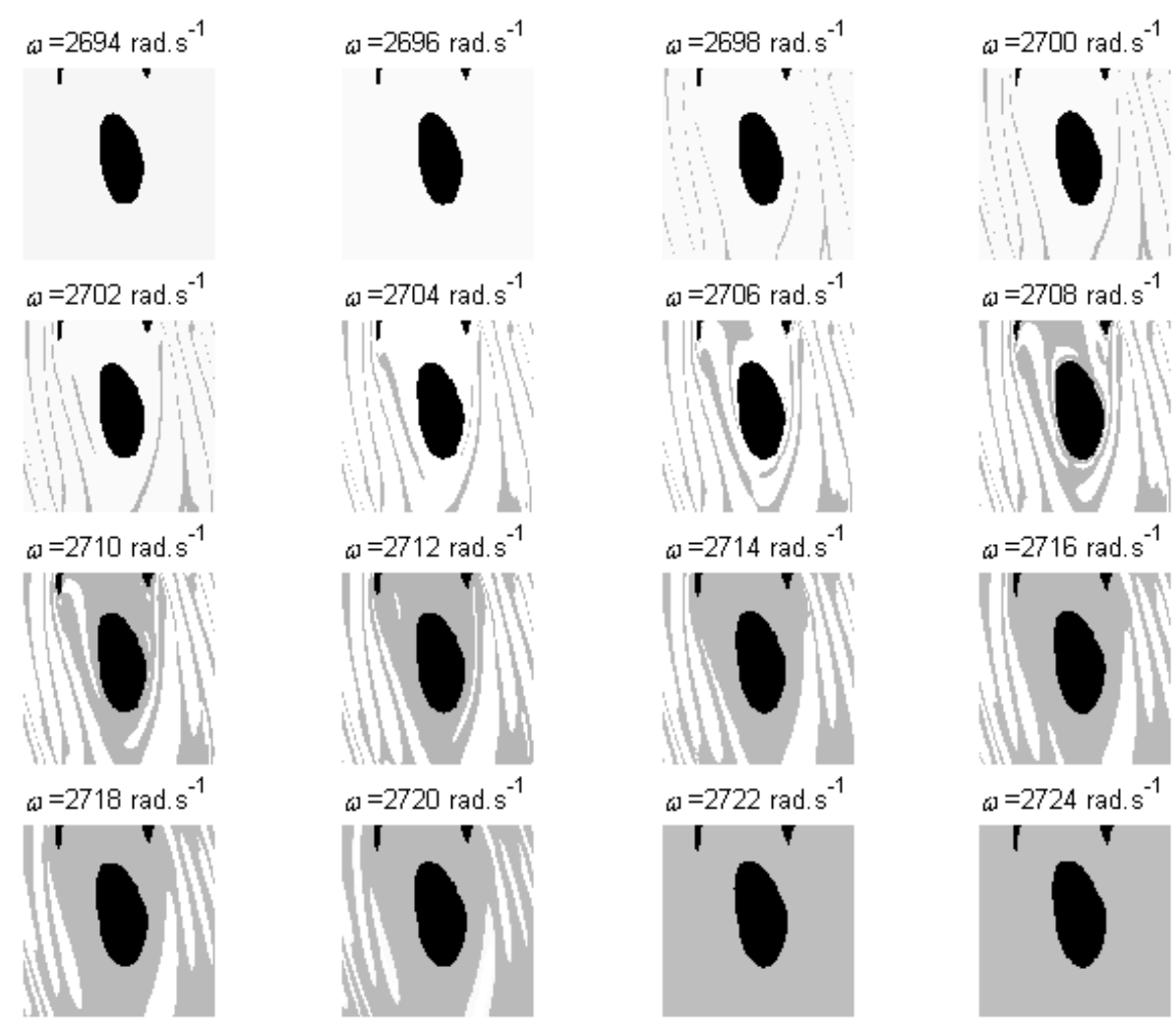

fig. 10: attraction basins in the $\left(a_{1}, a_{2}\right)$ space of the different oscillation states. The initial conditions are $\left(a_{1}, a_{2}\right) \in\left[-6 \times 10^{-9}, 6 \times 10^{-9}\right]^{2}$ and $a_{3}=0$.

For $\omega=2710 \mathrm{rad} . \mathrm{s}^{-1}$, the three possible attractors are plotted in fig. 11. 

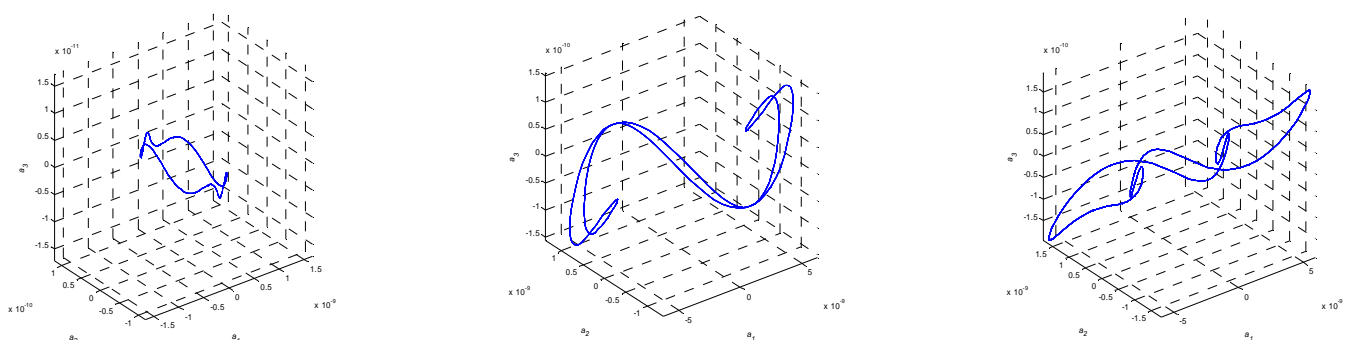

fig. 11: phase portrait of the three possible attractors at $\omega=2710 \mathrm{rad} \cdot \mathrm{s}^{-1}$ in the $\left(a_{1}, a_{2}, a_{3}\right)$ space.

\section{Comparison with experimental data}

In [13], the authors propose simple formulas for the pull-in voltage of simple microstructures, such as cantilever beams or circular diaphragms. These formulas are partly modelbased and partly experiment-based, through the use of fitting parameters whose values are established after a series of tests. Osterberg gives, for the pull-in voltage of circular plates without residual stress:

$V_{p i} \approx 1.51 \sqrt{\frac{E h^{3} G^{3}}{\left(1-v^{2}\right) \varepsilon_{0} R_{0}^{4}}}$

where $G$ is the electrostatic gap and $\varepsilon_{0}$ is the permittivity of vacuum.

Since (28) has been established for small displacements, it should not be valid for $G \gg>h$. We use our model and a plane-capacitance approximation of the electrostatic pressure to determine the pull-in voltage for a constant value of product $h . G$. The following set of parameters are used (from [13]): $R_{0}=10^{-3} \mathrm{~m}, h_{0}=14.4 \times 10^{-6} \mathrm{~m}, G_{0}=10^{-6} \mathrm{~m}, E=169 \mathrm{GPa}, v=0.3$, $\rho=2320 \mathrm{~kg} \cdot \mathrm{m}^{-3}$. The damping coefficient is high enough so that there is no overshooting ${ }^{3}$ of the pull-in limit (i.e. the system is critically damped): in this geometry, there can be no dynamic pull-in phenomenon. A shooting method is then used to determine the value of the pull-in voltage.

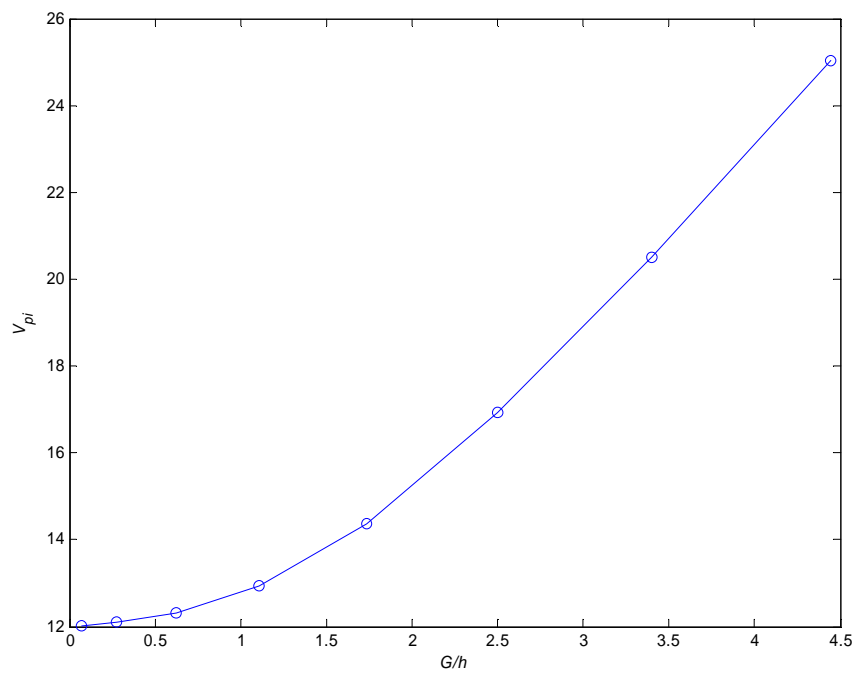

fig. 12: value of the pull-in voltage as the gap-to-thickness ratio increases. Because of the plate's stiffening, the calculated voltage becomes much larger than the expected $11.95 \mathrm{~V}$.

We then introduce parameter $\alpha$ so that the actual gap and beam thickness are respectively: $G=\alpha G_{0}(29)$

and

\footnotetext{
${ }^{3}$ This can be shown by an analysis of the Reynolds equation for thin films [7].
} 
$h=\frac{1}{\alpha} h_{0}$

The results obtained for different values of parameter $\alpha$ are shown in fig. 10. These results have to be compared with the nominal value of $V_{p i}=11.95 \mathrm{~V}$ obtained with (28). The curve in fig. 12 shows that, for increasing values of $\alpha$, even though the plate's thickness decreases, it becomes more difficult to pull in. The cause of this phenomenon is that, as $\alpha$ increases, the internal stresses of the beam are no longer dominated by bending but by the tensile stresses that build-up as the beam is being deflected.

\section{Conclusion}

In this paper, we have presented a method for modelling the large-displacement deflection of simple micro-structures, using an approach based on the modal analysis of the equations of Von Karman. This approach was illustrated in the case of a clamped circular diaphragm, which leads to analytical expressions of the mode shapes of the displacements and of the Airy stress function. Details concerning the implementation of this model were then given, as well as different results, as a basis for comparison with other modelling approaches. A method for deriving analytical solutions to several nonlinear problems was presented and used for validating the model from a mathematical point of view in the case of the dynamic excitation of a plate in the presence of possibly very nonlinear, "squeezed-film"-like damping. Finally, the results obtained with our model in the case of electrostatic actuation were compared to and showed good consistency with those of [13]: our model also could predict the increase in the pull-in voltage as the ratio gap/thickness increases.

Finally, this approach can be easily extended to other problems of structural dynamics, such as the actuation of beams and plates of various shapes as well as to other linear or nonlinear PDEs of MEMS literature, such as the equations governing squeezed-film damping or thermal actuation.

\section{References}

[1] M.H. Bao, "Micro mechanical transducers: pressure sensors, accelerometers and gyroscopes", Handbook of Sensors and Actuators, vol. 8, Elsevier, Amsterdam, 2000

[2] G.T.A. Kovacs, "Micromachined Transducers Sourcebook", McGraw-Hill, Inc., New York, NY, 1998

[3] E.M. Abdel-Rahman, A.H. Nayfeh, "Secondary resonances of electrically actuated resonant microsensors", Journal of Micromechanics and Microengineering, vol. 13, pp. 491501, 2003

[4] E.M. Abdel-Rahman et al., "Characterization of the mechanical behaviour of an electrically actuated microbeam", Journal of Micromechanics and Microengineering, vol. 12, pp. 759-766, 2002

[5] Q. Wang, W.H. Ko, "Modeling of touch mode capacitive sensors and diaphragms", Sensors and Actuators A, vol. 75, pp. 230-241, 1999

[6] G.W. Vogl, A.H. Nayfeh, "A reduced-order model for electrically actuated clamped circular plates", Proceedings of DETC'03, Chicago, Illinois, USA, September 2-6, 2003

[7] T. Veijola et al., "Equivalent circuit model of the squeezed gas film in a silicon accelerometer", Sensors and Actuators A, vol. 48, pp. 239-248, 1995

[8] L. Landau, E. Lifchitz, "Théorie de l'élasticité”, Mir, Moscow, 1967

[9] C.M. Harris, A.G. Piersol, "Harris' shock and vibration handbook", McGraw-Hill, Inc., New York, NY, 2002

[10] M. Géradin, D. Rixen, "Mechanical vibrations. Theory and application to structural dynamics", Wiley and Sons, Chichester, 1994

[11] A.H. Nayfeh, D.T. Mook, "Nonlinear oscillations", Wiley and Sons, New York, 1979 
[12] L.F. Shampine, M.W. Reichelt, "The MATLAB ODE Suite", SIAM Journal on Scientific Computing, Vol. 18, pp. 1-22, 1997

[13] P.M. Osterberg, S.D. Senturia, "M-TEST: a test chip for MEMS material property measurement using electrostatically actuated test structures", Journal of Microelectromechanical Systems, vol. 6, n² 2, pp. 107-118, 1997

[14] E. W. Weisstein. "Basin of Attraction", MathWorld--A Wolfram Web Resource. http://mathworld.wolfram.com/BasinofAttraction.html

[15] D. Zwillinger, "Handbook of differential equations", Academic Press, Inc., San Diego, CA, 1992

[16] J. Juillard, M. Cristescu, "An inverse approach to the design of adaptive micro-mirrors", Journal of Micromechanics and Microengineering, vol 14, issue 3, pp. 347-355, 2004 